\title{
Effectiveness and safety of neuroablation for severe and treatment-resistant obsessive-compulsive disorder: a systematic review and meta-analysis
}

\author{
Yijie Lai, MD*; Tao Wang, MD*; Chencheng Zhang, MD, PhD; Guozhen Lin, MD; \\ Valerie Voon, MD, PhD; Jinwoo Chang, MD, PhD; Bomin Sun, MD, PhD
}

\begin{abstract}
Background: Several neuroablative procedures are available for severe and treatment-resistant obsessive-compulsive disorder (OCD), but limited knowledge about their relative clinical advantages and disadvantages poses obstacles for treatment decision-making. Methods: We searched PubMed, Embase, Scopus, Web of Knowledge and the Cochrane Library for reports up to February 2019. We reviewed the literature on the effectiveness (assessed using the Yale-Brown Obsessive Compulsive Scale [Y-BOCS]) and safety of various neuroablative interventions for severe and treatment-resistant OCD. Results: We included 23 studies involving 487 patients in the systematic review; 21 studies with 459 patients were included in the meta-analysis. Overall, neuroablation achieved a response rate (proportion of patients with $\geq 35 \%$ reduction in Y-BOCS) of 55\%. Most of the adverse events (88.4\%) were mild and transient. The top 3 adverse events were headache $(14.9 \%)$, cognitive deficits $(9.1 \%)$ and behaviour problems $(8.1 \%)$. Severe or permanent adverse events included personality changes $(2.3 \%)$ and brain edema or brain cyst $(1.5 \%)$. The response rates associated with capsulotomy, limbic leucotomy and cingulotomy were $59 \%$ (95\% confidence interval [Cl] 54-65), 47\% (95\% Cl 23-72) and 36\% (95\% Cl 23-50), respectively. Interventions with different coverages of the dorsal part of the internal capsule were associated with different adverse-event profiles but were unlikely to modify clinical effectiveness. Limitations: The level of evidence of most included studies was relatively low. Conclusion: Ablative surgeries are safe and effective for a large proportion of patients with severe and treatment-resistant OCD. Among the available procedures, capsulotomy seemed to be the most effective. Further research is needed to improve clinical effectiveness and minimize risks.
\end{abstract}

\section{Introduction}

Obsessive-compulsive disorder (OCD) is the fourth most common mental disorder, with a lifetime prevalence of $1 \%$ to $3 \%$. Patients with OCD are characterized by recurrent intrusive thoughts (obsessions), unwanted repetitive behaviours (compulsions) or most commonly by both types of clinical symptoms. ${ }^{1}$ Obsessive-compulsive disorder was ranked among the top 10 most handicapping conditions in terms of decreased quality of life. The disorder has also been identified as a leading global cause of nonfatal illness by the World Health Organization. ${ }^{2,3}$ People with OCD often experience impairments in social and occupational functioning. ${ }^{3}$ Firstline treatments for OCD involve cognitive behavioural therapy with exposure and response prevention, and pharmacotherapy with serotonin reuptake inhibitors. ${ }^{4}$ Although most patients experience adequate relief from OCD symptoms with cognitive behavioural therapy and medication, about
$30 \%$ to $40 \%$ of affected patients fail to respond to these treatment modalities. ${ }^{5,6}$

Neurosurgical interventions have long been used as a last resort for rare cases of severe and treatment-resistant OCD in many medical centres. ${ }^{1,7}$ Since the first published report of prefrontal leucotomy for "obsessional neurosis" in 1947, 8 ablative surgery has been explored as a valuable neurosurgical treatment option for OCD. Recent meta-analyses have suggested that neuroablation is more effective for OCD than its psychiatric neurosurgery counterpart, deep brain stimulation (DBS). ${ }^{8-10}$ The clinical use of ablative interventions is based on the assumption that these procedures can resolve dysfunction in cortico-striato-thalamo-cortical circuitry, which is believed to underlie the pathophysiology of OCD and response to treatment, as evidenced by electrophysiological and functional neuroimaging studies. ${ }^{11}$

Surgical studies of patients with severe and treatmentresistant OCD have employed 4 main neuroablative

Correspondence to: C. Zhang, Department of Functional Neurosurgery, Ruijin Hospital, No. 197, Second Ruijin Rd., Shanghai, 200025, China; i@cczhang.org

*These authors contributed equally to this work.

Submitted Apr. 14, 2019; Revised Aug. 5, 2019; Revised Oct. 25, 2019; Revised Dec. 24, 2019; Accepted Jan. 16, 2020; Published online June 17, 2020

DOI: 10/1503/jpn.190079 
procedures, which target different brain structures and networks: anterior capsulotomy (anterior limb of the internal capsule [ALIC]), cingulotomy (anterior cingulate cortex and the fibres of the cingulum), subcaudate tractotomy (frontothalamic fibres) and limbic leucotomy (a combination of anterior cingulotomy and subcaudate tractotomy). ${ }^{1}$ The clinical utility of these procedures has been examined separately in different studies. However, there exists no original study to date comparing the safety and clinical effectiveness (assessed using the standard Yale-Brown Obsessive Compulsive Scale [Y-BOCS $]^{12}$ ) of these different neuroablative procedures with each other. ${ }^{13}$ This gap in the literature makes it difficult for clinicians and patients to make an informed decision about which neuroablative intervention to choose. At present, specific treatment decisions are still based largely on individual hospitals' expertise and experience in performing a certain neuroablative procedure. No data are available to support the application of one procedure over another in terms of its effectiveness or safety profile. ${ }^{14}$

In this study, we aimed to gain a better understanding of the relative clinical advantages and disadvantages of the various neuroablative interventions used for severe and treatment-resistant OCD. We conducted a systematic review and meta-analysis of the reported clinical benefits and risks of the different neuroablative interventions for OCD. We also explored whether interventions that differentially covered areas of the internal capsule were associated with different clinical outcomes. The study results should provide a better understanding of the effectiveness and safety of the main neuroablative interventions currently available for severe and otherwise treatment-resistant OCD.

\section{Methods}

\section{Search strategy}

We performed a literature search using the following databases (from inception to February 2019): PubMed, Embase, Scopus, Web of Knowledge and the Cochrane Library. The MESH terms (PubMed), EMTREE terms (Embase) and key words (others) we used were as follows: "obsessive-compulsive disorder," "stereotactic," "ablation," "psychosurgery," "capsulotomy," "cingulotomy" and "tractotomy." We also reviewed the reference lists of retrieved articles and reviews to find additional relevant studies.

\section{Selection criteria}

The inclusion criteria applied to the records retrieved were as follows: original human studies assessing the effectiveness of neuroablative surgery for OCD; treatment effectiveness measured using the Y-BOCS in terms of response rate (responders, partial responders and nonresponders were defined as showing reductions $\geq 35 \%, 35 \%$ to $25 \%$, and $<25 \%$, respectively, in total Y-BOCS score at final follow-up compared with baseline $)^{15}$ or the absolute or relative (\%) change in total Y-BOCS score from baseline to final follow-up; and written in English.
Exclusion criteria were as follows: animal studies, literature reviews, comments or correspondences, conference abstracts, patents, case reports or case series involving fewer than 3 patients; a lack of either pre- or postoperative Y-BOCS data; reports on the same patients at multiple follow-up assessments (in these cases, only the report with the final or most recent follow-up was included); results involving combinations of neuroablation and DBS; and a lack of information about the exact neuroablative procedure used (e.g., mentioning only "psychosurgery" or "psychiatric neurosurgery" as the intervention).

\section{Data extraction}

Two investigators (Y.L. and T.W.) independently extracted the following information from the included studies: study characteristics (OCD diagnostic criteria, inclusion and exclusion criteria and clinical outcome measures); sample size and demographic characteristics (sex, age at surgery, age at onset of OCD, duration of OCD and duration of follow-up); surgical variables (target, lesion technique and size of lesion); response rate, which was used as the primary effectiveness measure in this review; percent or absolute change in total Y-BOCS score, as well as outcomes related to depression and anxiety, which served as secondary outcome measures; the number and nature of adverse effects, which were first classified as either "mild and transient" or "severe or permanent" and then categorized according to 4 main classes (surgeryrelated, neurologic, neurobehavioural and other [e.g., weight change]). We imputed a missing response-rate value at last follow-up in the study of Csigo and colleagues ${ }^{16}$ using the mean value of the studies included in our meta-analysis.

To explore whether interventions with different coverages of internal capsule areas were associated with different clinical outcomes, we visually checked the postsurgical MRI images presented in the publications to match the targets to the lesion and categories proposed by Miguel and colleagues in $2018 .{ }^{17}$ Based on the contour of the $20 \%$ isodose line, the authors defined 4 categories to describe different coverages of the internal capsule. In our meta-analysis, we were able to put patients into 3 subgroups based on the target description or postsurgical MRI images: T group, with lesion areas comparable to 3 isocentres covering the majority of the ALIC; D group, with lesion areas comparable to double shots covering the ventral third of the anterior capsule/ventral striatum; and $\mathrm{S}$ group, with lesion areas comparable to a single isocentre covering the ventral capsule.

\section{Data synthesis and meta-analysis}

We used weighted-proportion analysis to adjust for study size using the DerSimonian-Laird model, taking the estimate of heterogeneity from the inverse-variance fixed-effect model. We constructed a forest plot to show study-level estimates, pooled estimates across studies (and 95\% confidence intervals [CIs]) and the relative weighted contribution of each study. We assessed heterogeneity using $Q$ statistics and the $I^{2}$ index; values of $p<0.1$ for the former and 
$>35 \%$ for the latter were deemed to be indicative of between-study heterogeneity. We used subgroup analyses to assess the effect of surgical covariates on the effectiveness of treatment. Because the majority of the included studies were observational and noncomparative, direct statistical comparisons between groups were inappropriate; therefore, we combined individual study results only within their respective groups. We calculated effectiveness values for the following: subgroups with different neuroablation procedures, subgroups with different coverage of internal capsule areas (T, D and S) and subgroups with different numbers of repeat procedures (yes: $>80 \%$ of patients received repeat procedures; mixed: $20 \%$ to $80 \%$ of patients received repeat procedures; no: $<20 \%$ of patients received repeat procedures). We performed multivariate random-effects metaregression using aggregate-level data to analyze correlations between outcomes and covariates (mean last follow-up period, mean age at surgery, mean age at disease onset, mean duration of illness and mean preoperative total Y-BOCS score). We conducted Begg's test and funnel plot analysis to detect potential publication bias.

\section{Results}

\section{Literature search}

Figure 1 presents study selection and the reasons for study exclusion. Our initial search retrieved 3003 studies. After we removed duplicates and screened titles and abstracts, we kept 92 studies for full-text review. Finally, we included 23 studies with a total of 487 patients $^{16,18-39}$ in the review and 21 studies with a total of 459 patients $^{16,18-20,22,24-39}$ in the metaanalysis.

\section{Main characteristics of included studies}

We separated the data from 5 studies into 12 data sets based on the type of surgical technique used in their patient sample, ${ }^{21,31,32,34,36}$ resulting in 30 data sets from 23 included studies, and incorporating 487 patients and 562 procedures (combinations of different surgeries at a single operation time were counted as a single procedure). The main characteristics of the included studies are described in Table 1 and Appendix 1, Tables S1 and S2 (available at jpn.ca/190079-a1). Among the procedures, capsulotomy was performed most commonly (343 patients [70.4\%] involving 16 studies), followed by cingulotomy (62 patients [12.7\%] involving 3 studies; Table 2). Although subcaudate tractotomy was reported in several studies, these studies either used symptom severity measures other than Y-BOCS or documented the results mixed with the effects of other surgeries, ${ }^{40-44}$ so no subcaudate tractotomy study was included in this review. Radiofrequency, focused ultrasonography, mechanical and other ablative approaches were used for capsulotomy, but radiofrequency was the only approach used for ablation in other brain areas. For capsulotomy, radiosurgery was performed on 139 patients $(40.5 \%)$ in 8 studies, and radiofrequency ablation was performed on 174 patients $(50.7 \%)$ in 7 studies.
In 1 study, a considerable number of the procedures used for limbic leucotomy were conducted under the guidance of ventriculography. ${ }^{23}$ In another, stereotactic lesions were made under the guidance of high-pressure pneumoventriculography, but the resulting lesions comprised a vast coverage of brain structures, from the ventral striatum to dorsolateral frontal cortex. ${ }^{21}$ We excluded these 2 studies from further quantitative analysis, resulting in a total of 21 studies incorporating 459 patients. Across the 21 studies included in the meta-analysis, the mean duration of follow-up was 45.4 months (range 7.6-142.0).

\section{Treatment effectiveness}

\section{Overall and surgery-specific effectiveness}

The pooled response rate at last follow-up (mean duration 45.4 [range 7.6-142.0] months) was 55\% (95\% CI 50-61) for the whole population treated with neuroablation. Specifically, the observed response rates were 59\% (95\% CI 54-65), $36 \%$ (95\% CI 23-50) and 47\% (95\% CI 23-72) following capsulotomy, cingulotomy and limbic leucotomy, respectively. We found substantial heterogeneity $(p=0.010)$ between groups for the different surgeries, but the extent of the heterogeneity within each surgery group was small $\left(I^{2}<35 \%, p>0.1\right.$; Fig. 2). Sheth and colleagues ${ }^{34}$ examined 30 patients who underwent a repeated cingulotomy and/or an additional subcaudate tractotomy (resulting in a complete limbic leucotomy) after the failure of an initial cingulotomy. The response rate in this group was 53\% (95\% CI 34-72) at follow-up (mean $65.2 \pm 54.6$ months). Zhang and colleagues ${ }^{39}$ reported that a combination of cingulotomy and capsulotomy achieved a response rate of $71 \%$ (95\% CI 29-96) at 12-month follow-up. Based on the studies included in our metaanalysis (9 data sets, 6 studies, 124 patients, all capsulotomy), $18,20,24,27,28,31,32$ we calculated a response rate of $47 \%(95 \%$ CI 37-56) at 12-month follow-up. No significant publication bias was present in the included studies, according to funnel plot analysis and Begg's test ( $p=0.28$; Appendix 1, Fig. S3).

The pooled total Y-BOCS score decreased from 33.1 (95\% CI 33.0-33.3) at baseline to 19.3 (95\% CI 18.9-19.7) at last follow-up (mean duration 45.4 [range 7.6-142.0] months; Fig. 3). At 12-month follow-up, the pooled Y-BOCS score (reported in 14 studies involving 337 patients) was 18.5 (95\% CI 17.8-19.2). The Y-BOCS scores at 12-month follow-up for patients who underwent capsulotomy (10 studies, 249 patients) or cingulotomy (2 studies, 51 patients) were $17.5(95 \%$ CI 16.8-18.3) and 21.7 (95\% CI 19.3-24.0), respectively. The reductions in Y-BOCS scores at 12 months (52 patients) and last follow-up (mean duration 40.6 months, 180 patients) were 12.6 (95\% CI 11.5-13.7) and 13.6 (95\% CI 12.5-14.7; Appendix 1 , Fig. S4). The pooled percent reductions in the total Y-BOCS score at 12 months (109 patients) and last follow-up (mean duration 48.0 months, 198 patients) were $32.20 \%$ (95\% CI 28.33-36.07) and 43.55\% (95\% CI 41.87-45.22; Appendix 1, Fig. S5). Specifically, the pooled reductions in Y-BOCS scores at last follow-up were $46.13 \%$ (95\% CI 40.53-51.73) for the capsulotomy group (104 patients; $\left.I^{2}=0.0 \% ; p=0.54\right), 32.20 \%(95 \%$ CI 18.98-45.42) for the limbic leucotomy group (17 patients; 
$\left.I^{2}=0.0 \% ; p=0.18\right)$, and $24.26 \%(95 \%$ CI $16.08-32.44)$ for the cingulotomy group (43 patients; $I^{2}=45.6 \% ; p=0.18$ ).

\section{Target-specific effectiveness in capsulotomy}

We also categorized patients with lesions in the internal capsule into 3 groups (, D and $S$ ) according to the site and na- ture of their lesions based on the postsurgical MRI, as outlined earlier. ${ }^{17}$ We excluded 1 study from this analysis because the original data for specific lesions in the internal capsule were not reported..$^{20}$ Results for the unilateral lesions reported by Ruck and colleagues ${ }^{32}$ were also excluded. Most included studies involved patients belonging to $\mathrm{T}$ group

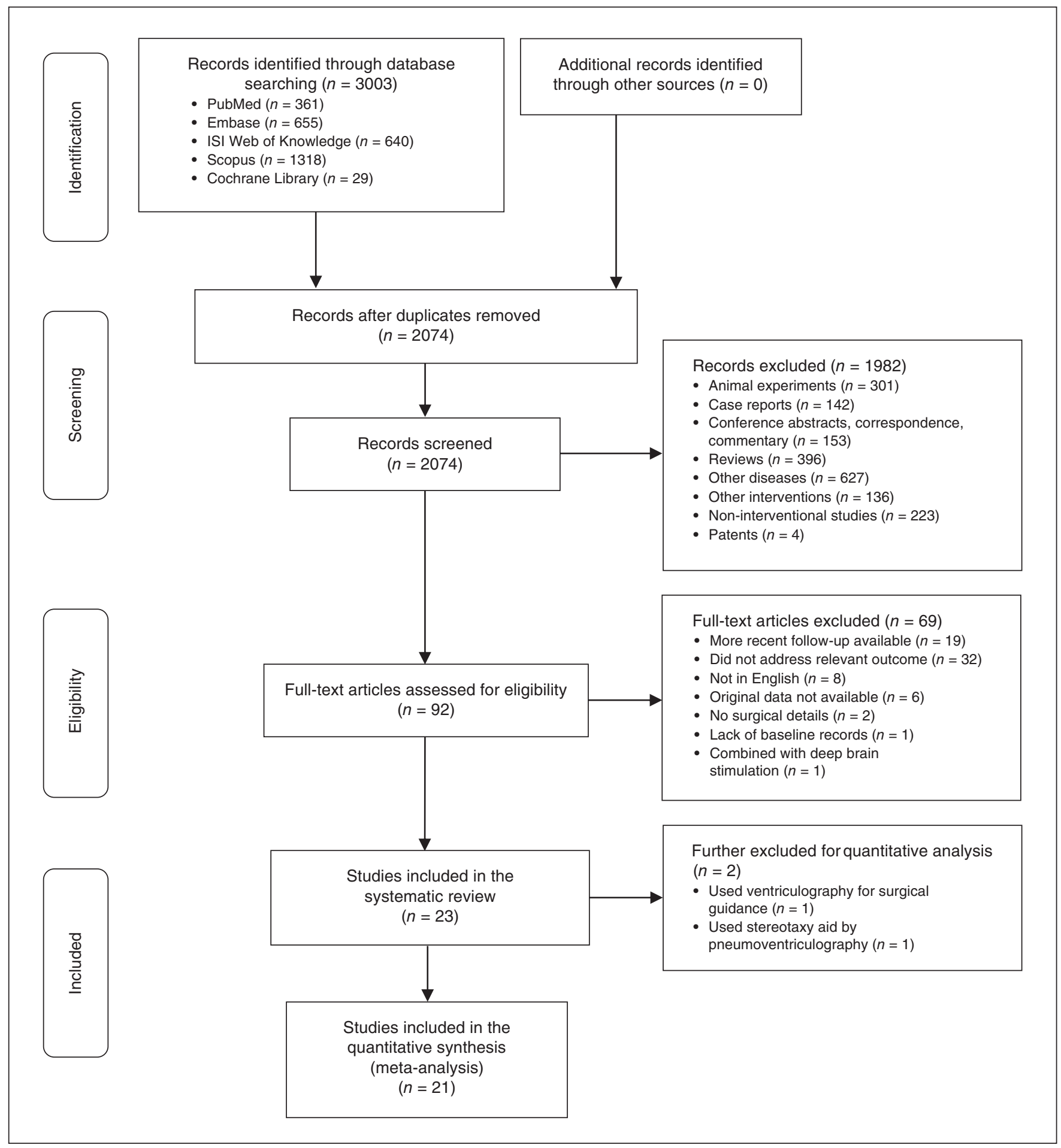

Fig. 1: Flow diagram for study selection. 


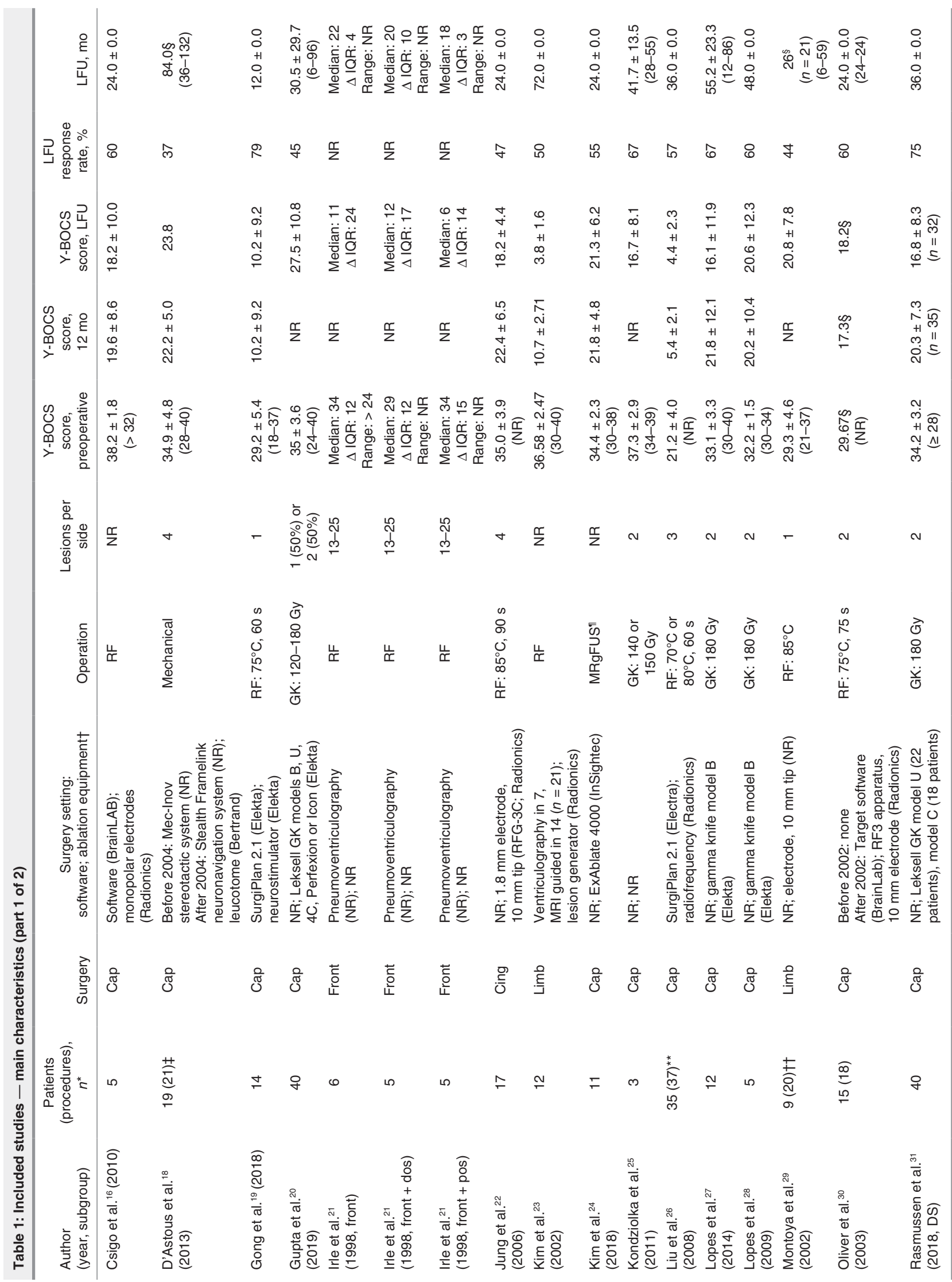




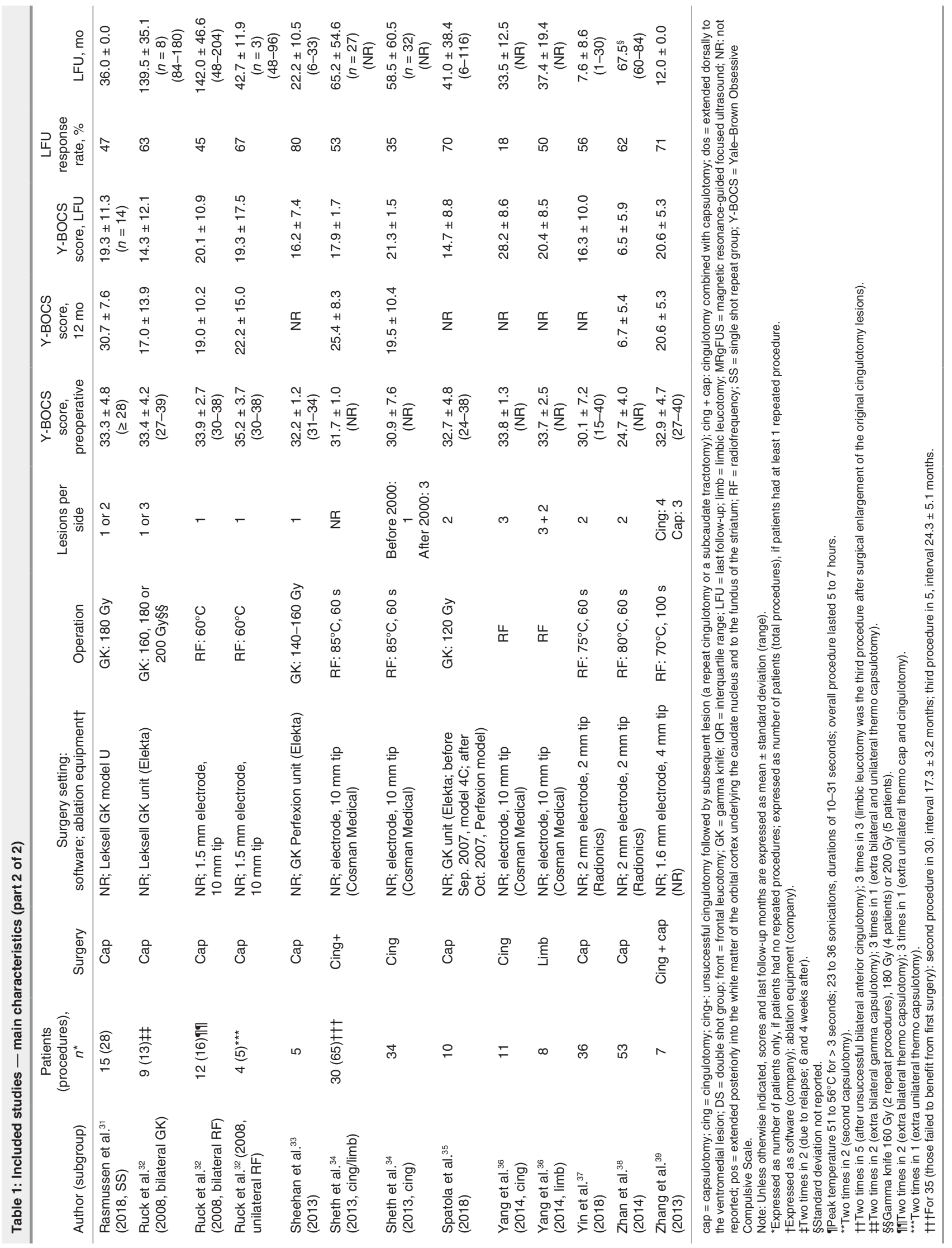




\begin{tabular}{|c|c|c|}
\hline Item & $\begin{array}{l}\text { Patients, } n \\
\quad(\%)\end{array}$ & $\begin{array}{l}\text { Datasets } \\
\text { (studies), } n\end{array}$ \\
\hline \multicolumn{3}{|l|}{ Surgical procedure $(n=487)$} \\
\hline Capsulotomy & $343(70.43)$ & $19(16)$ \\
\hline Cingulotomy & $62(12.73)$ & $3(3)$ \\
\hline Cingulotomy with subsequent lesions* & $30(6.16)$ & $1(1)$ \\
\hline Cingulotomy with capsulotomy & $7(1.44)$ & $1(1)$ \\
\hline Limbic leucotomy & $29(5.95)$ & $3(3)$ \\
\hline Frontal leucotomy & $16(3.29)$ & $3(1)$ \\
\hline \multicolumn{3}{|l|}{ Ablative technique (capsulotomy) $(n=343)$} \\
\hline Radiofrequency & $174(50.73)$ & $8(7)$ \\
\hline Radiosurgery & $139(40.52)$ & $9(8)$ \\
\hline Focused ultrasound & $11(3.21)$ & $1(1)$ \\
\hline Mechanical lesion & $19(5.54)$ & $1(1)$ \\
\hline \multicolumn{3}{|l|}{ Repeat procedure $(n=487) \dagger$} \\
\hline Yes & $54(11.09)$ & $3(3)$ \\
\hline Mixed & $25(5.13)$ & $3(1)$ \\
\hline No & $408(83.78)$ & $24(21)$ \\
\hline \multicolumn{3}{|c|}{$\begin{array}{l}{ }^{*} \text { Repeated cingulotomy or subcaudate tractotomy. } \\
\dagger Y e s=>80 \% \text { received a repeat procedure; mixed }=\text { approximately } 20 \% \text { to } 80 \% \\
\text { received a repeat procedure; no }=<20 \% \text { received a repeat procedure. }\end{array}$} \\
\hline
\end{tabular}

(8 studies, 9 data sets, 186 patients) and D group (6 studies, 7 data sets, 96 patients). We identified only 5 patients who belonged to $\mathrm{S}$ group. Response rates were 58\% (95\% CI 50-65), $67 \%$ (95\% CI 56-77), and 80\% (95\% CI 28-99) for groups T, D and S, respectively (Fig. 4). We noted no significant heterogeneity between groups for response rate $(p=0.33)$, absolute reduction in total Y-BOCS score $(p=0.72)$ or percent reduction in total Y-BOCS score ( $p=0.92$; Appendix 1, Figs. S6 and S7). Rasmussen and colleagues ${ }^{31}$ examined patients with bilateral single-shot lesions, located centrally in the capsule (defined as an "original gamma knife capsulotomy target" by Miguel and colleagues $^{17}$ ). At an average follow-up of $9.0 \pm 0.4$ months after the initial single-shot procedure, only 1 patient (of the 15 examined) met the criteria for a full or partial responder. ${ }^{31}$ These patients subsequently received a repeat single-shot lesion located ventrally to the first-stage shot, which eventually turned the lesion into a D group type of lesion.

Additionally, the pooled response rates in studies with more than $80 \%$ of patients who received repeat procedures (yes group), studies with $20 \%$ to $80 \%$ of patients with repeat procedures (mixed group) and studies with less than $20 \%$ of patients receiving repeat procedures (no group) were $50 \%$ (95\% CI 36-64), 55\% (95\% CI 31-77) and 56\% (95\% CI 49$63)$, respectively. We found no significant heterogeneity between groups with different proportions of repeat procedures ( $p=0.75$; Appendix 1, Fig. S8).

\section{Effectiveness and demographic variables}

We observed no significant correlations between demographic variables (age of OCD onset, duration of OCD, age at surgery, preoperative Y-BOCS score, duration of followup) and outcome measures (response rate and absolute and percent reduction in total Y-BOCS score) at final follow-up (Appendix 1, Table S9).

\section{Depression and anxiety}

Among the 21 studies included in the meta-analysis, 15 (involving 19 data sets) contained outcome data on symptoms of depression (in 365 patients) and anxiety (in 286 patients) following ablative surgery (Appendix 1, Table S10). Depression symptoms were assessed using the Hamilton Depression Rating Scale, the Beck Depression Inventory or the Montgomery-Åsberg Depression Rating Scale. ${ }^{45-47}$ Anxiety symptoms were measured using the Hamilton Anxiety Rating Scale, the Beck Anxiety Inventory, the Brief Scale of Anxiety, or the State-Trait Anxiety Inventory. ${ }^{48-51}$ After ablative surgery, 14 data sets incorporating 310 patients (85\%) reported significant improvement in depression symptoms from baseline. Likewise, 11 data sets incorporating 247 patients $(86 \%)$ reported decreased anxiety following surgery.

\section{Treatment safety}

\section{Overall adverse effects}

Adverse effects (either presence or absence) were documented in 18 studies involving 395 patients $(86 \%$ of the population included in the meta-analysis); 3 studies provided no information about adverse effects. One study was excluded because the reported adverse effects in OCD patients were mixed with results from patients whose primary diagnoses were not OCD; 2 other studies were excluded because information about adverse effects was not reported. ${ }^{29,36,37}$ Of the 395 patients in the adverse effects analysis, 307 were treated with capsulotomy, 17 with cingulotomy, 64 with cingulotomy or limbic leucotomy, and 7 with cingulotomy combined with capsulotomy. Only 7 data sets involved active surveillance for adverse effects, using the Systematic Assessment for Treatment Emergent Effects event list or the Execution, Apathy, and Disinhibition scale. ${ }^{27,28,31,32}$ The incidence and nature of the reported adverse effects for each data set (or study) are summarized in Appendix 1, Table S11. Overall, across the different ablative procedures, most of the documented adverse effects ( $88.4 \%$ of all adverse effects) were transient and manageable. The top 3 most frequently reported mild and transient adverse effects were postoperative headache (incidence $14.9 \%$ ), cognitive deficits $(9.1 \%)$ and behaviour problems (8.1\%). Severe or permanent adverse effects were as follows: personality changes $(2.3 \%)$, brain cysts or brain edema $(1.5 \%)$, behaviour disorders $(1.3 \%)$, weight change $(1.0 \%)$, cognitive deficits $(1.0 \%)$, urinary incontinence $(0.5 \%)$, suicide $(0.5 \%)$, seizure $(0.5 \%)$, subdural empyema $(0.3 \%)$, intracerebral hemorrhage $(0.3 \%)$, pulmonary embolus $(0.3 \%)$, hemiplegia $(0.3 \%)$, disturbances of consciousness $(0.3 \%)$ and headache $(0.3 \%)$. Overall, the median incidence of mild and transient adverse effects was $1.0 \%$ (range 0.3-14.9). The median incidence of severe or permanent adverse effects was $0.5 \%$ (range $0.3-2.3$ ).

\section{Surgery-specific adverse effects}

We found only 1 study that reported on the incidence of adverse effects specifically following cingulotomy (3 of 17 patients showed memory problems lasting less than 2 months). ${ }^{22}$ Another study reported adverse effects 
collapsed across patients with cingulotomy and patients with limbic leucotomy. ${ }^{34}$ In our analysis, we considered the adverse effects of cingulotomy and limbic leucotomy together as the adverse effects of limbic system surgery ( $n=81$ for limbic system surgery). ${ }^{34,36}$ Table 3 presents the results of this analysis. The median incidence of mild and transient effects was $1.7 \%$ (range $0.0-19.2$ ) in the capsulotomy group and $0.0 \%$ (range 0.0-9.9) in the limbic system surgery group. The median incidence rates of severe or permanent adverse effects were $0.3 \%$ (range $0.0-2.9$ ) in the capsulotomy group and $0.0 \%$ (range $0.0-2.5$ ) in the limbic system surgery group.

Two cases of suicide were documented after cingulotomy: 1 patient had a history of comorbid major depressive disor- der (preoperative Beck Depression Inventory score 41) and the other patient had a history of comorbid bipolar disorder and presented with depression (Beck Depression Inventory score 39) at the time of surgery..$^{34}$ Additionally, Montoya and colleagues $^{29}$ reported a case of suicide after limbic leucotomy. In all 3 deaths, the patients exhibited suicidal ideation before surgery and had previously attempted suicide. In frontal leucotomy, Irle and colleagues ${ }^{21}$ reported that 8 patients $(50 \%$ of all patients) with extended lesions in the ventral striatum developed alcohol dependence $(n=6)$ or alcohol and benzodiazepine dependence $(n=2)$. These researchers also found that extension of the ventromedial frontal lesion to the dorsolateral prefrontal cortex was associated with a lower

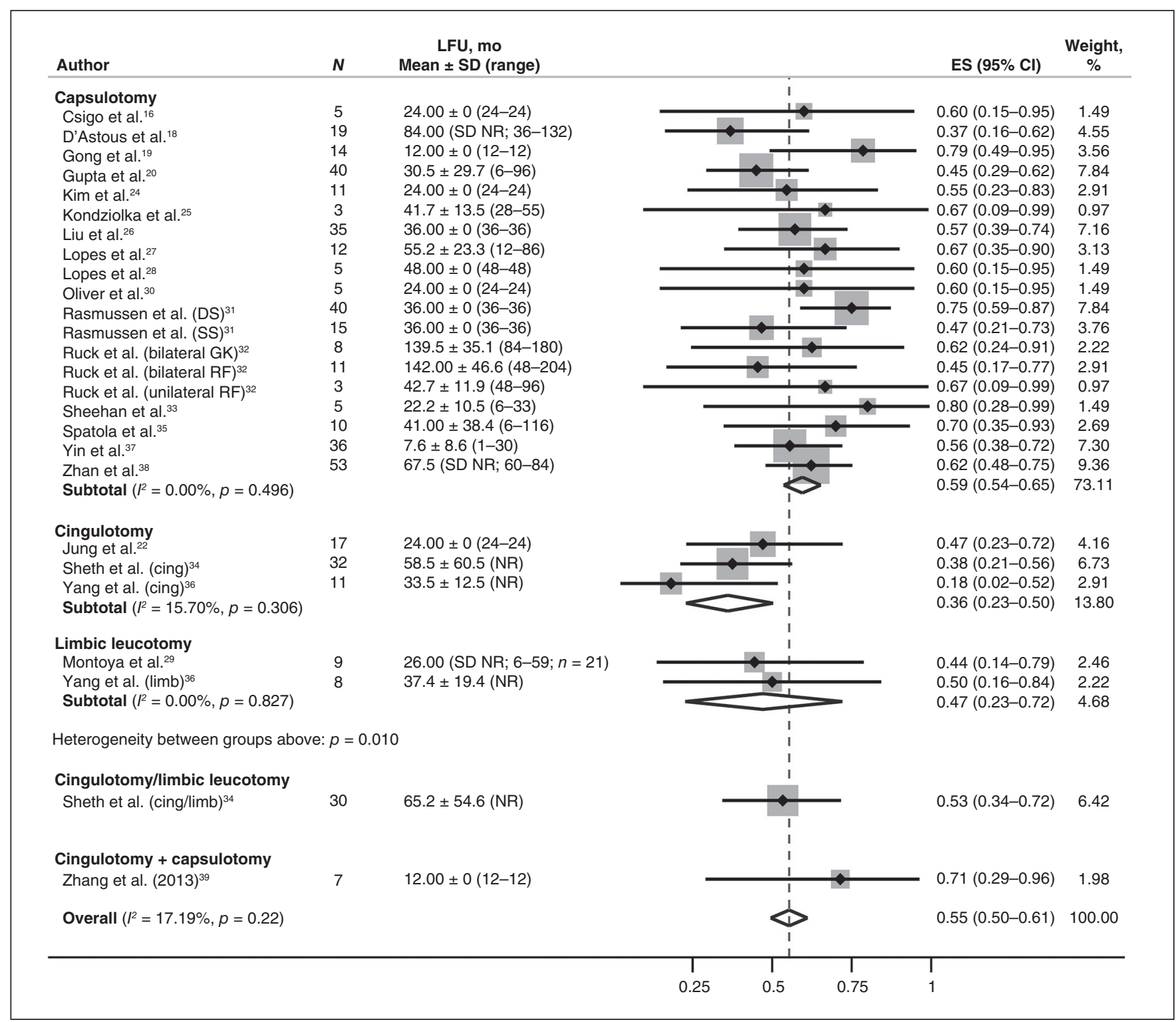

Fig. 2: Response rate at last follow-up after different neuroablation procedures. The diamond at the bottom is the pooled mean and its $95 \%$ confidence interval $(\mathrm{Cl})$ under a random-effects model. Last follow-up is expressed as mean \pm standard deviation $(\mathrm{SD})$ and range. cing $=\mathrm{cin}$ gulotomy; DS = double shot group; ES = estimated value for response rate; GK = gamma knife; LFU = last follow-up; limb = limbic leucotomy; $\mathrm{NR}=$ not reported; $\mathrm{RF}$ = radiofrequency; $\mathrm{SS}$ = single shot repeat group. 
performance IQ after surgery. However, the latter finding remains to be explored further, given that the result was based on data from only 5 participants. ${ }^{21}$

\section{Target-specific adverse effects in capsulotomy}

As described earlier, ${ }^{17}$ we categorized patients who received capsulotomy into 3 groups (T, D and S). The percentages of patients in these groups with reported adverse effects (presence or absence) were $81 \%$ (150 patients), 100\% (96 patients) and $100 \%$ (5 patients), respectively. However, the latter observation was based on only 1 study that included 5 patients. ${ }^{33}$ Therefore, we examined only the adverse event profiles for patient groups $\mathrm{T}$ and $\mathrm{D}$. For these groups, the median incidence of reported severe or permanent adverse effects were $1.3 \%$ (range $0.0-6.0$ ) and $0.0 \%$ (range $0.0-3.1$ ), respectively. The respective group median incidence rates of mild and transient adverse effects were $0.7 \%$ (range $0-17.3$ ) and $1.0 \%$ (range 0-58.3; Appendix 1, Table S12).

In some studies, patients' psychiatric-neurosurgical and neurologic adverse effects, such as delirium, confabulation

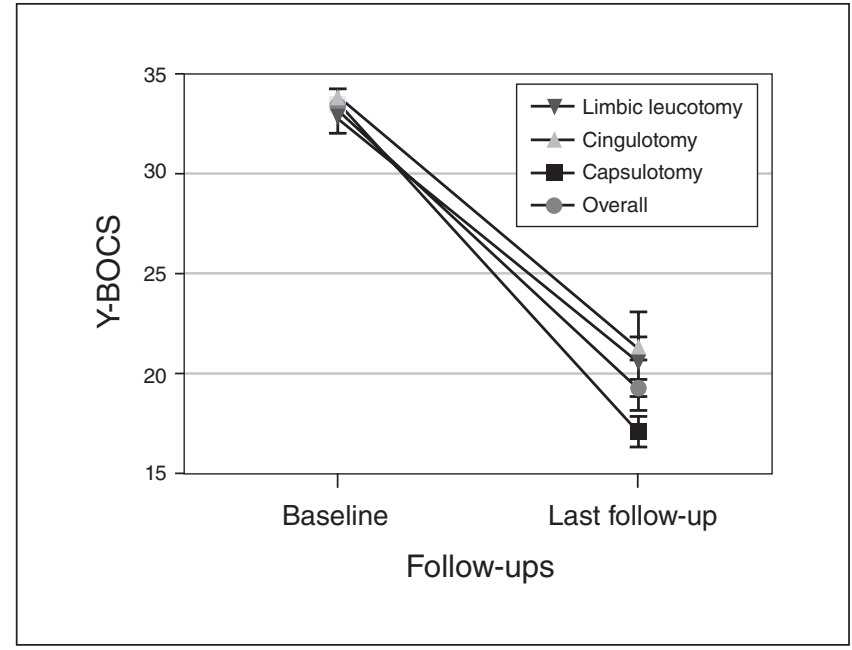

Fig. 3: Overall Yale-Brown Obsessive Compulsive Scale (Y-BOCS) scales at baseline and last follow-up; scales are plotted as pooled estimated values. Bars indicate $95 \%$ confidence intervals.

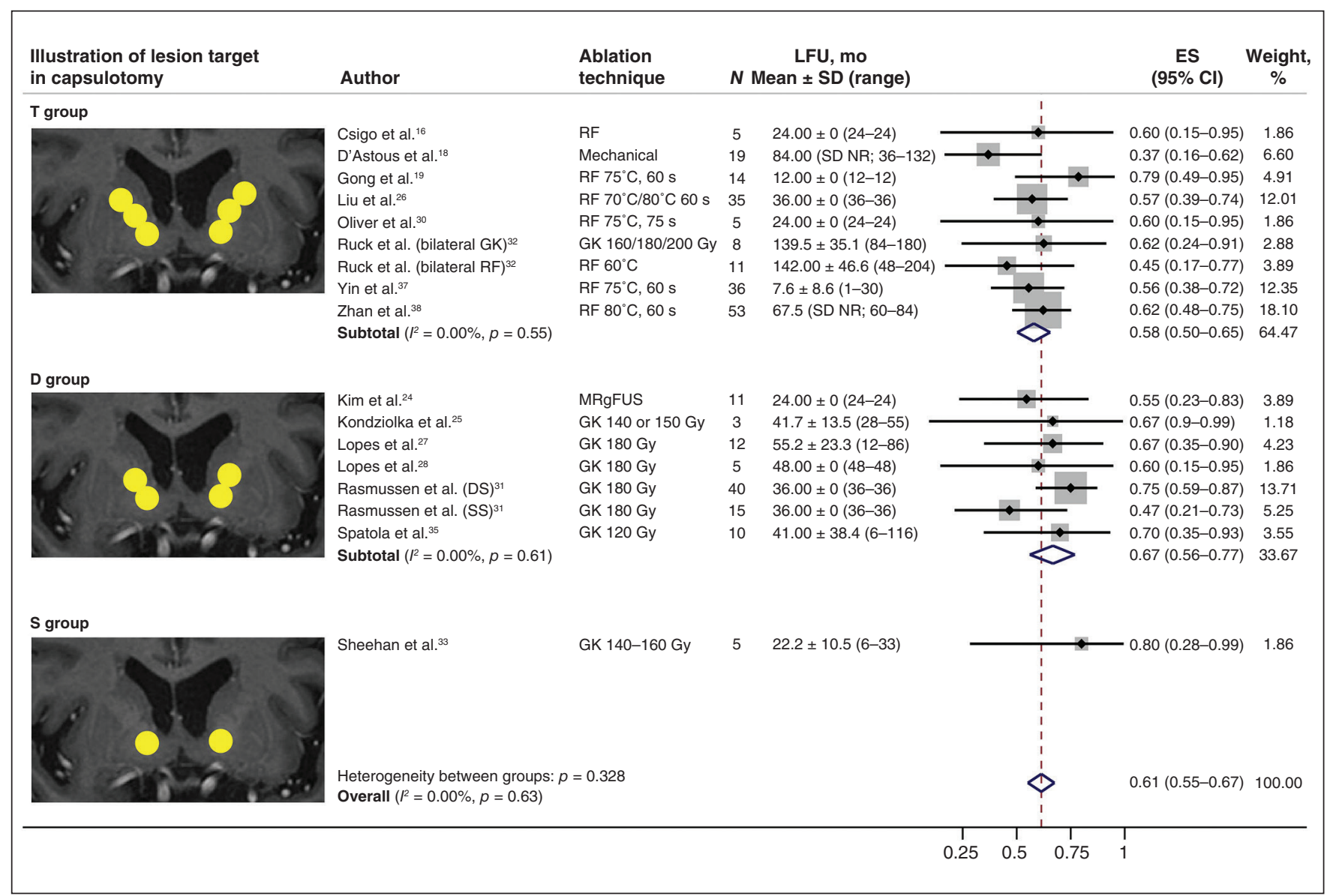

Fig. 4: Response rates at last follow-up in patient groups with different coverages of internal capsule areas (T, D and S). T group: lesion comparable to 3 isocentres covering the majority of the anterior limb of the internal capsule; $\mathrm{D}$ group: lesion comparable to double shots covering the ventral third of the anterior capsule/ventral striatum; S group: lesion comparable to a single isocentre covering the ventral capsule. Yellow circles indicate the areas of the lesion in the coronal plane. Last follow-up is expressed as mean \pm standard deviation (SD) and range. $\mathrm{Cl}=$ confidence interval; DS = double shot group; ES = estimated value for response rate; GK = gamma knife; LFU = last follow-up; MRgFUS = magnetic resonance-guided focused ultrasound; NR = not reported; RF = radiofrequency; SS = single shot repeat group. 
Table 3: Adverse effects in capsulotomy and limbic system surgery

\begin{tabular}{|c|c|c|}
\hline Adverse effect & Capsulotomy, \% & Limbic system surgery, \% \\
\hline \multicolumn{3}{|l|}{ Surgery-related } \\
\hline \multicolumn{3}{|l|}{ Severe or permanent } \\
\hline Brain edema or cyst & 1.6 & 1.2 \\
\hline Intracerebral hemorrhage & 0.3 & 0.0 \\
\hline Subdural empyema & 0.0 & 1.2 \\
\hline \multicolumn{3}{|l|}{ Mild and transient } \\
\hline Brain edema or cyst & 2.6 & 0.0 \\
\hline Asymptomatic lacunar infarct & 2.0 & 0.0 \\
\hline Skin edema & 1.3 & 0.0 \\
\hline Deep vein thrombosis & 1.0 & 0.0 \\
\hline Fever & 1.0 & 0.0 \\
\hline Intracerebral hemorrhage & 1.0 & 0.0 \\
\hline Pneumonia & 0.3 & 0.0 \\
\hline Sore throat & 0.3 & 0.0 \\
\hline Urinary infection & 0.3 & 0.0 \\
\hline \multicolumn{3}{|l|}{ Neurological } \\
\hline \multicolumn{3}{|l|}{ Severe or permanent } \\
\hline Cognitive deficits & 1.3 & 0.0 \\
\hline Urinary incontinence & 0.7 & 0.0 \\
\hline Hemiplegia & 0.3 & 0.0 \\
\hline Seizure & 0.3 & 1.2 \\
\hline Disturbances of consciousness & 0.3 & 0.0 \\
\hline Headache & 0.3 & 0.0 \\
\hline \multicolumn{3}{|l|}{ Mild and transient } \\
\hline Headache & 19.2 & 0.0 \\
\hline Cognitive deficits & 9.1 & 9.9 \\
\hline Vestibular symptoms & 9.1 & 0.0 \\
\hline Urinary incontinence & 6.2 & 1.2 \\
\hline Disturbances of consciousness & 4.9 & 0.0 \\
\hline Paresthesia & 2.9 & 0.0 \\
\hline Hiccup & 2.3 & 0.0 \\
\hline Vertigo & 1.3 & 0.0 \\
\hline Abdominal discomfort & 1.0 & 0.0 \\
\hline Seizure & 0.3 & 4.9 \\
\hline Urinary retention & 0.0 & 2.5 \\
\hline Sialorrhea & 0.3 & 0.0 \\
\hline Muscle pain & 0.3 & 0.0 \\
\hline \multicolumn{3}{|l|}{ Neurobehavioural } \\
\hline \multicolumn{3}{|l|}{ Severe or permanent } \\
\hline Personality changes & 2.9 & 0.0 \\
\hline Behaviour disorder* & 1.6 & 0.0 \\
\hline Suicide & 0.0 & 2.5 \\
\hline \multicolumn{3}{|l|}{ Mild and transient } \\
\hline Behaviour disorder ${ }^{\star}$ & 10.4 & 0.0 \\
\hline Mood changest & 6.5 & 0.0 \\
\hline Personality changes & 2.0 & 4.9 \\
\hline \multicolumn{3}{|l|}{ Other } \\
\hline \multicolumn{3}{|l|}{ Severe or permanent } \\
\hline Weight change & 1.6 & 1.2 \\
\hline \multicolumn{3}{|l|}{ Mild and transient } \\
\hline Weight change & 5.9 & 0.0 \\
\hline \multicolumn{3}{|l|}{ Median incidence (range) } \\
\hline Severe or permanent & $0.3(0.0-2.9)$ & $0.0(0.0-2.5)$ \\
\hline Mild and transient & $1.7(0.0-19.2)$ & $0.0(0.0-9.9)$ \\
\hline \multicolumn{3}{|c|}{$\begin{array}{l}\text { Capsulotomy group, } n=307 \text {; limbic system surgery group, } n=81 \text {. } \\
\text { *Including sexual disinhibition, insomnia, increased appetite, hypersomia, somnolence, slurred } \\
\text { speech and childish behaviour. } \\
\text { †Including depression, anxiety and mania. }\end{array}$} \\
\hline
\end{tabular}

and visual hallucinations, were attributed to radiationinduced perilesional edema or radiation necrosis, which responded to corticosteroids within a few days. ${ }^{27,32}$ Radiation-induced severe adverse effects, including cyst formation, were reported in 2 studies: 1 study $^{20}$ involved a patient who underwent gamma ventral capsulotomy after a failed cingulotomy procedure (equipment unknown), and the other study ${ }^{31}$ included 3 patients who underwent gamma ventral capsulotomy with the model $\mathrm{C}$ gamma knife (maximum 180 Gy). One of the patients in the latter study developed radionecrosis with extensive edema requiring surgical intervention. ${ }^{31}$

\section{Discussion}

This systematic review and meta-analysis indicated that neuroablation is a relatively effective and safe intervention for rare cases of severe and treatment-resistant OCD. In the following sections, we summarize the results supporting this general conclusion and discuss the clinical implications, along with addressing current challenges and suggesting directions for future research.

\section{Treatment effectiveness}

The overall response rate across different neuroablative procedures was $55 \%$ at a mean follow-up of 45.4 months (range 7.6-142). Among the 3 main neuroablative procedures reviewed, the response rate associated with capsulotomy $(59 \%)$ was higher than the response rates seen with limbic leucotomy (47\%) and cingulotomy (36\%). The response rate data were substantiated by using the Y-BOCS as a continuous measure of clinical response. The mean percent reductions in total Y-BOCS score were $46 \%$ in the capsulotomy group, $32 \%$ in the limbic leucotomy group and $24 \%$ in the cingulotomy group. Based on these results, we tentatively concluded that each ablative procedure was able to produce a clinically meaningful improvement in the core symptoms of OCD. Moreover, the response rate with capsulotomy may have been higher than the rates seen with cingulotomy and limbic leucotomy, although this result could not be statistically confirmed because of a lack of comparative studies. ${ }^{13}$ Both conclusions should be further qualified, however, because large interindividual patient differences typically exist in clinical response to ablative surgery: some patients show significant clinical improvements, and other patients experience little or no symptomatic relief after surgery.

Additionally, improvement in the clinical symptoms of OCD after ablative surgery was already evident at 12-month follow-up. Although the response rate to capsulotomy at 12 -month follow-up was $47 \%$, which appeared to be inferior to the observed response rate of $59 \%$ at last follow-up, we did not observe a significant correlation between the duration of follow-up and clinical outcome measures, and this was in line with 
the results of 2 previous systematic reviews. ${ }^{14,52}$ Although it has been suggested that specific ablation techniques can be relevant to the response trend (e.g., gamma knife lesions in white matter, which develop gradually over a few weeks or months, delaying the clinical effect), the therapeutic effects of neuroablative interventions resulted from functional interruption; reorganization would occur and would largely stabilize in some patients within the first year after surgery. ${ }^{24,31,32,34}$ Therefore, it seems unlikely that the ablative procedures reviewed here would take several years to exhibit their therapeutic effects. Also, it is conceivable that the symptoms of nonresponders improved in the first year of treatment, but failed to maintain this initial speed of recovery. ${ }^{18}$ Unfortunately, the overall database is currently limited and did not allow us to draw any firm conclusions about the timing of the clinical response in the first year after ablative surgery.

This meta-analysis substantiated and extended the results of 2 previous systematic reviews on the effectiveness and safety of neuroablation for severe and treatment-resistant OCD. ${ }^{14,52}$ One of these reviews, which included 2 studies of anterior cingulotomy (81 patients) and 8 studies of anterior capsulotomy (112 patients), reached the conclusion that both ablative procedures were effective. ${ }^{14}$ However, this review was limited by the fact that it examined a relatively small number of studies and patients. In the other review, the focus was on contrasting ablative surgery to DBS; little attention was given to the different ablative procedures used in the studies. ${ }^{52}$ Moreover, the authors provided no information about the clinical effects of lesions in different parts of the internal capsule.

In this review, we observed that capsulotomy was the most commonly performed ablative surgery (about $70 \%$ of all patients received this procedure; $n=343$ ). We further categorized patients with lesions in the internal capsule into 3 groups (T, D and S) based on target descriptions and postsurgical MRI scans. ${ }^{17}$ In this analysis, patients had documented lesions of the ventral portion of the capsule, but the lesion coverage of the dorsal parts of the capsule varied between groups. The observed response rates at last follow-up were $58 \%$ for group T, $67 \%$ for group D and $80 \%$ for group S. However, the observation in group $S$ was based on only 1 study with 5 patients. ${ }^{33}$ Based on the results we obtained from groups $\mathrm{T}$ and $\mathrm{D}$, extending the lesion dorsally in the internal capsule did not seem to significantly affect clinical effectiveness. In a recent study, patients with OCD received bilateral single-shot radiosurgery targeting the midpoint of the ALIC, conceivably focusing on fibres connecting the medial and orbitofrontal cortices, the head of the caudate and the midline thalamus. ${ }^{31}$ However, the observed response rate following this procedure was only $7 \%$ (1 responder of 15 patients). In contrast, a repeat single-shot procedure or a double-shot procedure (D group), both targeting the capsule more ventrally (targeting the longitudinal fibre tracts that connect the anterior cingulate and orbitofrontal projections to the striatum, thalamus and brainstem), were associated with a significantly higher response rate $(75 \%$; 30 responders of 40 patients). ${ }^{31}$ Although these results appear to indicate that the specific target in the ALIC could be a key to improving clinical outcomes, we could not rule out the possibility that the higher response rate in the single-shot repeat group was a delayed effect of radiosurgery, because the single-shot group was followed for only 9 months, not 12 months or longer. ${ }^{31}$

Our findings lent support to the view that the clinical effectiveness of capsulotomy for OCD is closely linked to lesions of the ventral portion of the ALIC (in the coronal plane), approximately near the posterior putaminal border (in the axial plane) ${ }^{17}$ Anatomically, the ventral segment of the ALIC, embedded within the ventral striatum and anterior commissure, is the region that carries fibres from the ventromedial prefrontal cortex and the orbital frontal cortex, whose dysfunction has long been implicated in the pathophysiology of OCD. ${ }^{28,36}$ In contrast, researchers have distinguished 4 dorsal components of the ALIC, which carry fibres of ventrolateral prefrontal cortex, dorsolateral prefrontal cortex, dorsal anterior cingulate cortex and medial prefrontal cortex. ${ }^{17}$ The action of capsulotomy on OCD symptom severity could stem primarily from its effect on ventral capsule targets, thereby affecting brain functional networks involving the orbitofrontal cortex and ventromedial prefrontal cortex..$^{28,36}$ In this context, restricting lesion areas to the ventral capsule could be promising for reducing adverse effects without compromising therapeutic effects. It has also been hypothesized that the pathophysiological substrates of OCD, at least in some patients, are not symmetric, but instead are confined to 1 hemisphere of the brain. ${ }^{6,32}$ If this hypothesis is valid, unilateral rather than bilateral capsulotomy can be used to alleviate OCD symptoms while reducing the risk of adverse effects. To date, however, only 1 study involving 4 patients has explored this therapeutic approach to OCD treatment. ${ }^{32}$

\section{Treatment safety}

Each ablative surgery was associated with risks of adverse effects. Overall, most of the documented adverse effects (about 90\%) were transient and manageable. The most commonly reported mild and transient adverse event was headache, followed by cognitive deficits and behaviour problems. Of note, a relatively high incidence of headache was reported by Rasmussen and colleagues. ${ }^{31}$ These researchers observed that most patients who experienced headache had received gamma knife surgery. It seems plausible that the application of a relatively high peak dose of 180 to $200 \mathrm{~Gy}$ could have contributed to patients' postsurgical headaches observed in this and other gamma knife studies. ${ }^{20,27,28,31}$ It may also help to explain patients' vestibular adverse effects (nausea and vomiting). ${ }^{27,31}$ Indeed, studies using lower doses (e.g., 120, 140 or $150 \mathrm{~Gy}$ ) have not reported such adverse effects. $25,33,35$

Capsulotomy was associated with higher incidence of mild and transient adverse effects than limbic system surgery. However, this finding could have been confounded by differences in the study methods used to assess adverse effects. Whereas limbic system lesion studies employed a more general questioning method to elicit patient-reported effects, 4 capsulotomy studies used active surveillance to ascertain 
the occurrence of adverse effects..$^{27,28,31,32}$ Active surveillance (e.g., Systematic Assessment for Treatment Emergent Effects) is more sensitive for detecting subtle discomfort such as headache or paresthesia, and should be encouraged in studies to establish the full adverse effect profile associated with each ablative procedure. Accordingly, it remains to be determined whether the 2 ablative procedures genuinely differed from each other in incidence of mild and transient adverse effects.

The incidence of severe or permanent adverse effects was relatively low and did not differ greatly between capsulotomy and limbic system surgery. Notwithstanding their low incidence, more enduring adverse effects included personality changes $(2.3 \%)$, as well as brain cysts or brain edema $(1.5 \%)$, posing a health threat to patients. Although some of the enduring and serious adverse effects could be attributed to risks inherent in the medical procedure, others might be preventable by altering the size, exact location or nature of the therapeutic lesion. Indeed, the modification of currently used ablative interventions, as well as the development of novel, more sophisticated surgical techniques, would likely help prevent or reduce the incidence of both transient and enduring adverse effects. A possible barrier to reaching this goal remains a relative lack of knowledge about the precise causes and mechanisms of the adverse effects associated with ablative surgery.

We observed that the clinical effectiveness of capsulotomy for patient groups $\mathrm{T}$ and $\mathrm{D}$ was relatively similar. Furthermore, the incidence rates of mild and transient adverse effects were comparable between the groups (median: $\mathrm{T}$ group $0.7 \%$; D group $1.0 \%$ ), but the nature of the adverse effect profiles differed between the groups. Group differences present in the incidence rates of severe or permanent adverse effects (median: T group 1.3\%; D group 0.0\%): patients in the $\mathrm{T}$ group showed personality changes and cognitive deficits more often. Early capsulotomies for treating OCD used up to 3 isocentres (T group) to cover the majority of the ALIC. Subsequently, it was argued that reducing the extension of the lesions might help increase effectiveness and limit the risk of adverse effects. ${ }^{32}$ Unfortunately, no standard definition exists for the ventral portion of ALIC. As well, no consensus exists on whether ventral capsulotomy is superior to traditional capsulotomy. Although these results suggest that the MRI-based postsurgical lesion subtyping outlined by Miguel and colleagues ${ }^{17}$ has clinical utility, it should be noted that the specific ablation technique used (e.g., radiofrequency ablation, radiosurgery, gamma knife, magnetic resonance-guided focused ultrasound) also differed between groups $\mathrm{T}$ and $\mathrm{D}$. In any case, it would be worthwhile to determine whether restricting the lesion area to the ventral capsule is associated with fewer adverse effects while maintaining or even improving the clinical effectiveness of the intervention. ${ }^{17,32}$

\section{Challenges and future directions}

This review indicated that ablative surgical procedures can offer a relatively safe and effective intervention for severe and treatment-resistant OCD, but the evidence also showed that a substantial portion of patients failed to respond clinically to these interventions. Clinicians faced with this challenge have sometimes performed an additional or repeat ablative surgical intervention in an effort to alleviate the patient's OCD symptoms. In 1 study, for example, over half of the nonresponders after an initial cingulotomy showed a good clinical response to subsequent lesion interventions, including a repeat cingulotomy and/or an additional subcaudate tractotomy. ${ }^{34}$ However, the responder status (full, partial or nonresponse) was similar $(p=0.24)$ between patients who underwent a single cingulotomy $(n=30)$ and those who underwent subsequent procedures $(n=27) .{ }^{34}$ Therefore, we cannot rule out the possibility that the earlier surgery exerted a latent effect in these patients, because they were followed for only $10.2 \pm 1.7$ months after the initial cingulotomy before the second procedure was conducted. As well, patients who receive multiple procedures have a higher risk of adverse effects, including apathy and executive dysfunction, than patients who receive a single surgery. ${ }^{32}$ Finally, it is important to ensure that patients receive adequate care and therapeutic interventions after ablative surgery. In particular, behavioural therapies (such as cognitive behavioural therapy with exposure and response prevention) can offer additional clinical benefits to patients after surgical treatment. ${ }^{31,53}$

Another approach to improving clinical effectiveness is to search for predictors of treatment response or prognostic biomarkers. In the present review, we explored whether certain demographic and clinical characteristics of patients could serve as a predictor of clinical response. However, the results demonstrated that none of the variables examined had predictive validity. To reduce heterogeneity within patient groups and predict treatment response, a pathophysiologybased approach to finding predictors could be more successful, employing measures of altered brain function and structure implicated in OCD. For example, an MRI study found that the clinical response of patients who underwent cingulotomy could be accurately predicted from measures of anterior cingulate cortex structure and connectivity. ${ }^{6}$ The identification of genetic or epigenetic markers is also a promising approach to clinical response prediction and personalized medicine, because OCD is likely an etiologically and genetically heterogeneous disorder. ${ }^{4}$

Ablative surgery has been used for decades. So far, however, DBS (specifically, DBS of the ventral capsule or ventral striatum) is the only surgical intervention that has received approval from the United States Food and Drug Administration for OCD treatment. ${ }^{54}$ Indeed, reversibility and adjustability represent major advantages of DBS. However, the relatively high cost of DBS could be a problem for patients and health care providers. Other significant problems that may occur in DBS treatment involve battery failure, lead migration and device misplacement or malfunction. ${ }^{55,56}$ In contrast, neuroablation is relatively cost-effective and has a long-lasting clinical effect, rendering patients free of the inconvenience of battery changes and equipment maintenance. Emerging new techniques, including focused ultrasound and laser ablation, are pushing the field of ablative surgery forward in terms of both effectiveness and safety. ${ }^{55}$ 


\section{Limitations}

This review had several limitations. First, the level of evidence of most included studies was relatively low, limiting our ability to draw firm conclusions. Most studies were observational in nature and did not adopt a comparative research design. We identified only 1 randomized controlled trial, ${ }^{27}$ but its small sample size $(n=16)$ precluded extensive inferences. It should be noted that although randomized controlled trials are the standard of excellence for treatment studies, it is usually not ethical to assign a treatment (compared to another treatment, no treatment or placebo) by randomization rather than by patient and physician choice. The major concern is that the patient's health could be compromised by withholding appropriate treatment for the duration of the study.

Second, as we suggested earlier, the identification of adverse effects in most studies was based on passive surveillance. This method may have failed to detect subtle psychiatric symptoms and signs following ablative surgery, such as mood changes and suicidal ideation. This problem may have been particularly relevant when unstructured psychiatric interviews were conducted rather than formal systematic clinical assessments. ${ }^{24}$ Therefore, we could have underestimated the incidence of mild transient adverse effects in this review. However, an important issue that requires clarification is whether some of the reported adverse effects are linked to the neuroablative intervention itself and/or to the natural course of OCD or its comorbidity with other psychiatric disorders. ${ }^{20}$

\section{Conclusion}

Based on this review, we have tentatively drawn a number of conclusions about the effectiveness and safety of neuroablation for severe and treatment-resistant OCD.

Each ablative surgical procedure can provide a relatively effective intervention for the primary symptoms of OCD. In most cases, ablative procedures can also alleviate symptoms of comorbid depression and anxiety disorders. Capsulotomy seems to be superior to limbic leucotomy and cingulotomy in terms of clinical effectiveness.

Each ablative surgery carries risks of adverse effects, which are mostly transient and manageable. In very rare cases, adverse effects are more enduring and pose a serious health concern. Lesion subtyping in capsulotomy offers a promising approach to reducing adverse effects while seems not to affect effectiveness.

Finally, it should be emphasized that neuroablation is an appropriate treatment option for only rare cases of severe and treatment-resistant OCD. In addition, patient assessment and neurosurgical procedures should be carried out only at expert medical centres. The highest priorities for future research are to improve the clinical effectiveness and to minimize the risks of ablative surgical interventions.

Acknowledgements: The authors appreciated the critical comments from Dianyou Li, Department of Functional Neurosurgery, Ruijin Hospital, Shanghai Jiao Tong University School of Medicine, and
Haiyan Jin, Department of Psychiatry, Ruijin Hospital, Shanghai Jiao Tong University School of Medicine, in preparation of the manuscript.

Affiliations: From the Department of Functional Neurosurgery, Ruijin Hospital, Shanghai Jiao Tong University School of Medicine, Shanghai, China (Lai, Wang, Zhang, Sun); the Department of Psychiatry, Ruijin Hospital, Shanghai Jiao Tong University School of Medicine, Shanghai, China (Lin); the Department of Psychiatry, University of Cambridge, Cambridge, United Kingdom (Voon); and the Department of Neurosurgery, Yonsei University College of Medicine, Seoul, South Korea (Chang).

Funding: B. Sun is supported by a Natural Science Foundation of China Grant (81771482) and a Shanghai Science and Technology Commission International Cooperation Project (STCSM, NO:18410710400). V. Voon is supported by a Medical Research Council Senior Clinical Fellowship (MR/P008747/1).

Competing interests: None declared.

Contributors: Y.J. Lai, C. Zhang, V. Voon, J. Chang and B. Sun designed the study. Y.J. Lai, T. Wang and C. Zhang acquired the data, which Y.J. Lai, C. Zhang, G. Lin, J. Chang and B. Sun analyzed. Y.J. Lai and C. Zhang wrote the article, which all authors reviewed. All authors approved the final version to be published and can certify that no other individuals not listed as authors have made substantial contributions to the paper.

\section{References}

1. Hirschtritt ME, Bloch MH, Mathews CA. Obsessive-compulsive disorder: advances in diagnosis and treatment. JAMA 2017;317: 1358-67.

2. Grant JE. Clinical practice: obsessive-compulsive disorder. N Engl J Med 2014;371:646-53.

3. Veale D, Roberts A. Obsessive-compulsive disorder. BMJ 2014; 348:g2183.

4. Koran LM, Hanna GL, Hollander E, et al. Practice guideline for the treatment of patients with obsessive-compulsive disorder. Am J Psychiatry 2007;164(Suppl):5-53.

5. Atmaca M. Treatment-refractory obsessive compulsive disorder. Prog Neuropsychopharmacol Biol Psychiatry 2016;70:127-33.

6. Banks GP, Mikell CB, Youngerman BE, et al. Neuroanatomical characteristics associated with response to dorsal anterior cingulotomy for obsessive-compulsive disorder. JAMA Psychiatry 2015; 72:127-35.

7. Bari AA, Mikell CB, Abosch A, et al. Charting the road forward in psychiatric neurosurgery: proceedings of the 2016 American Society for Stereotactic and Functional Neurosurgery workshop on neuromodulation for psychiatric disorders. J Neurol Neurosurg Psychiatry 2018;89:886-96.

8. Dibden WA. Prefrontal leucotomy for obsessional neurosis: report of a case. Med J Aust 1947;2:511.

9. Lipsman N, Lozano AM. Obsessive-compulsive disorder. J Neurosurg 2013;118:489-90.

10. De Rojas JO, Saunders JA, Luminais C, et al. Electroencephalographic changes following direct current deep brain stimulation of auditory cortex: a new model for investigating neuromodulation. Neurosurgery 2013;72:267-75.

11. Whiteside SP, Port JD, Abramowitz JS. A meta-analysis of functional neuroimaging in obsessive-compulsive disorder. Psychiatry Res 2004;132:69-79.

12. Goodman WK, Price LH, Rasmussen SA, et al. The Yale-Brown Obsessive Compulsive Scale. I. Development, use, and reliability. Arch Gen Psychiatry 1989;46:1006-11.

13. Yavin D, Casha S, Wiebe S, et al. Lumbar fusion for degenerative disease: a systematic review and meta-analysis. Neurosurgery 2017;80:701-15

14. Brown LT, Mikell CB, Youngerman BE, et al. Dorsal anterior cingulotomy and anterior capsulotomy for severe, refractory obsessivecompulsive disorder: a systematic review of observational studies. J Neurosurg 2016;124:77-89.

15. Pallanti S, Quercioli L. Treatment-refractory obsessive-compulsive disorder: methodological issues, operational definitions and therapeutic lines. Prog Neuropsychopharmacol Biol Psychiatry 2006;30:400-12. 
16. Csigo K, Harsanyi A, Demeter G, et al. Long-term follow-up of patients with obsessive-compulsive disorder treated by anterior capsulotomy: a neuropsychological study. J Affect Disord 2010;126: 198-205.

17. Miguel EC, Lopes AC, McLaughlin NCR, et al. Evolution of gamma knife capsulotomy for intractable obsessive-compulsive disorder. Mol Psychiatry 2019;24:218-40.

18. D'Astous M, Cottin S, Roy M, et al. Bilateral stereotactic anterior capsulotomy for obsessive-compulsive disorder: long-term followup. J Neurol Neurosurg Psychiatry 2013;84:1208-13.

19. Gong F, Li P, Li B, et al. A study of cognitive function in treatmentrefractory obsessive-compulsive disorder treated with capsulotomy. J Neurosurg 2018;128:583-95.

20. Gupta A, Shepard MJ, Xu Z, et al. An international radiosurgery research foundation multicenter retrospective study of gamma ventral capsulotomy for obsessive compulsive disorder. Neurosurgery 2019;85:808-16.

21. Irle E, Exner C, Thielen $\mathrm{K}$, et al. Obsessive-compulsive disorder and ventromedial frontal lesions: clinical and neuropsychological findings. Am J Psychiatry 1998;155:255-63.

22. Jung $\mathrm{HH}$, $\mathrm{Kim} \mathrm{CH}$, Chang JH, et al. Bilateral anterior cingulotomy for refractory obsessive-compulsive disorder: long-term follow-up results. Stereotact Funct Neurosurg 2006;84:184-9.

23. Kim MC, Lee TK, Choi CR. Review of long-term results of stereotactic psychosurgery. Neurol Med Chir (Tokyo) 2002;42:365-71.

24. Kim SJ, Roh D, Jung HH, et al. A study of novel bilateral thermal capsulotomy with focused ultrasound for treatment-refractory obsessive-compulsive disorder: 2-year follow-up. J Psychiatry Neurosci 2018;43:327-37.

25. Kondziolka D, Flickinger JC, Hudak R. Results following gamma knife radiosurgical anterior capsulotomies for obsessive compulsive disorder. Neurosurgery 2011;68:28-33.

26. Liu K, Zhang H, Liu C, et al. Stereotactic treatment of refractory obsessive compulsive disorder by bilateral capsulotomy with 3 years follow-up. J Clin Neurosci 2008;15:622-629.

27. Lopes AC, Greenberg BD, Canteras MM, et al. Gamma ventral capsulotomy for obsessive-compulsive disorder: a randomized clinical trial. JAMA Psychiatry 2014;71:1066-76.

28. Lopes AC, Greenberg BD, Noren G, et al. Treatment of resistant obsessive-compulsive disorder with ventral capsular/ventral striatal gamma capsulotomy: a pilot prospective study. J Neuropsychiatry Clin Neurosci 2009;21:381-92.

29. Montoya A, Weiss AP, Price BH, et al. Magnetic resonance imagingguided stereotactic limbic leukotomy for treatment of intractable psychiatric disease. Neurosurgery 2002;50:1043-9, discussion 1049-52.

30. Oliver B, Gascon J, Aparicio A, et al. Bilateral anterior capsulotomy for refractory obsessive-compulsive disorders. Stereotact Funct Neurosurg 2003;81:90-5.

31. Rasmussen SA, Noren G, Greenberg BD, et al. Gamma ventral capsulotomy in intractable obsessive-compulsive disorder. Biol Psychiatry 2018:84:355-64

32. Ruck C, Karlsson A, Steele JD, et al. Capsulotomy for obsessivecompulsive disorder: long-term follow-up of 25 patients. Arch Gen Psychiatry 2008;65:914-21.

33. Sheehan JP, Patterson G, Schlesinger D, et al. Gamma knife surgery anterior capsulotomy for severe and refractory obsessive-compulsive disorder. J Neurosurg 2013;119:1112-8.

34. Sheth SA, Neal J, Tangherlini F, et al. Limbic system surgery for treatment-refractory obsessive-compulsive disorder: a prospective long-term follow-up of 64 patients. J Neurosurg 2013;118:491-7.

35. Spatola G, Martinez-Alvarez R, Martinez-Moreno N, et al. Results of gamma knife anterior capsulotomy for refractory obsessive- compulsive disorder: results in a series of 10 consecutive patients. J Neurosurg 2018;131:376-383.

36. Yang JC, Ginat DT, Dougherty DD, et al. Lesion analysis for cingulotomy and limbic leucotomy: comparison and correlation with clinical outcomes. J Neurosurg 2014;120:152-63.

37. Yin D, Zhang C, Lv Q, et al. Dissociable frontostriatal connectivity: mechanism and predictor of the clinical efficacy of capsulotomy in obsessive-compulsive disorder. Biol Psychiatry 2018;84:926-36.

38. Zhan S, Liu W, Li D, et al. Long-term follow-up of bilateral anterior capsulotomy in patients with refractory obsessive-compulsive disorder. Clin Neurol Neurosurg 2014;119:91-5.

39. Zhang QJ, Wang WH, Wei XP. Long-term efficacy of stereotactic bilateral anterior cingulotomy and bilateral anterior capsulotomy as a treatment for refractory obsessive-compulsive disorder. Stereotact Funct Neurosurg 2013;91:258-61.

40. Hodgkiss AD, Malizia AL, Bartlett JR, et al. Outcome after the psychosurgical operation of stereotactic subcaudate tractotomy, 19791991. J Neuropsychiatry Clin Neurosci 1995;7:230-4.

41. Taren JA, Curtis GC, Gebarski SS. Late local and remote structural changes after capsulotomy for obsessive compulsive disorder. Stereotact Funct Neurosurg 1994;63:1-6.

42. Goktepe EO, Young LB, Bridges PK. A further review of the results of stereotactic subcaudate tractotomy. Br J Psychiatry 1975;126:270-80.

43. Knight G. Further observations from an experience of 660 cases of stereotactic tractotomy. Postgrad Med J 1973;49:845-54.

44. Van Vliet IM, Van Well EPL, Bruggeman R, et al. An evaluation of irreversible psychosurgical treatment of patients with obsessivecompulsive disorder in the Netherlands, 2001-2008. J Nerv Ment Dis 2013;201:226-8.

45. Hamilton M. A rating scale for depression. J Neurol NeurosurgPsychiatry 1960;23:56-62.

46. Beck AT, Ward $\mathrm{CH}$, Mendelson $\mathrm{M}$, et al. An inventory for measuring depression. Arch Gen Psychiatry 1961;4:561-71.

47. Montgomery SA, Asberg M. A new depression scale designed to be sensitive to change. Br J Psychiatry 1979;134:382-9.

48. Hamilton M. The assessment of anxiety states by rating. Br J Med Psychol 1959;32:50-5.

49. Tyrer P, Owen RT, Cicchetti DV. The brief scale for anxiety: a subdivision of the comprehensive psychopathological rating scale. J Neurol NeurosurgPsychiatry 1984;47:970-5.

50. Beck AT, Epstein N, Brown G, et al. An inventory for measuring clinical anxiety: psychometric properties. J Consult Clin Psychol 1988; 56:893-7.

51. Marteau TM, Bekker H. The development of a six-item short-form of the state scale of the Spielberger State-Trait Anxiety Inventory (STAI). Br J Clin Psychol 1992;31:301-6.

52. Kumar KK, Appelboom G, Lamsam L, et al. Comparative effectiveness of neuroablation and deep brain stimulation for treatment-resistant obsessive-compulsive disorder: a meta-analytic study. J Neurol Neurosurg Psychiatry 2019;90:469-473.

53. Spofford CM, McLaughlin NC, Penzel F, et al. OCD behavior therapy before and after gamma ventral capsulotomy: case report. Neurocase 2014;20:42-5.

54. Fins JJ, Mayberg HS, Nuttin B, et al. Analysis and commentary: misuse of the FDA's humanitarian device exemption in deep brain stimulation for obsessive-compulsive disorder. Health Aff 2011;30:302-11.

55. Kumar KK, Bhati MT, Ravikumar VK, et al. MR-guided focused ultrasound versus radiofrequency capsulotomy for treatmentrefractory obsessive-compulsive disorder: a cost-effectiveness threshold analysis. Front Neurosci 2019;13:66.

56. Miocinovic S, Somayajula S, Chitnis S, et al. History, applications, and mechanisms of deep brain stimulation. JAMA Neurol 2013;70:163-71. 